\title{
ASPECTOS TÉCNICOS DA INTERPOSIÇÃO ILEAL COM GASTRECTOMIA VERTICAL COMO POSSÍVEL OPÇÃO AO TRATAMENTO DO DIABETES MELLITUS TIPO 2
}

\author{
Technical aspects of ileal interposition with sleeve gastrectomy as a possible option for the treatment of type 2
}

\author{
Aureo Ludovico de PAULA, Alessandro SILVA, AC. Carolina C.L de PAULA, Sérgio VENCIO, Alfredo HALPERN
}

Trabalho realizado no Hospital de Especialidades, Goiânia, GO, Brasil.

DESCRITORES - Obesidade. Diabetes mellitus. Cirurgia Bariátrica.

Correspondência:

Áureo Ludovico de Paula, e-mail adepaula@uol.com.br

Fonte de financiamento: não há Conflito de interesses: não há

Recebido para publicação: 15/12/2009 Aceito para publicação: 22/03/2010

HEADINGS - Obesity. Diabetes mellitus. Bariatric surgery.
RESUMO - Introdução - Há evidências que os melhores resultados em termos de resolução do diabetes mellitus tipo 2 (DM2) em pacientes obesos mórbidos são atingidos com as derivações biliopancreáticas, em especial o duodenal switch. Essas operações caracterizamse por redução do estômago através de gastrectomia e o rápido trânsito dos alimentos para o íleo distal, através de expressiva derivação de segmentos do intestino delgado. A idéia da realização da técnica aqui apresentada baseou-se nestes princípios. Método - Os procedimentos são habitualmente realizados por via laparoscópica após pneumoperitônio com 12 a $15 \mathrm{mmHg}$ e seis trocárteres. O paciente é inicialmente posicionado em proclive de $30^{\circ} \mathrm{com}$ o cirurgião à direita. Inicia-se a gastrectomia vertical tendo como referência anatômica a trifurcação distal do vago anterior. A desvascularização da grande curvatura é realizada e se estende até o ângulo esofagogástrico. Com um calibrador intra-gástrico de 20 $\mathrm{mm}$ posicionado ao longo da pequena curvatura gástrica inicia-se a ressecção gástrica no antro com grampeador linear e adicionalmente é feita uma sutura contínua. Para a realização da interposição ileal na altura do jejuno proximal o ângulo duodenojejunal é identificado e o jejuno seccionado 20 a $30 \mathrm{~cm}$ à jusante com grampeador linear. $\mathrm{O}$ ceco é identificado e o íleo distal seccionado a $30 \mathrm{~cm}$ no sentido proximal. Um segmento de 150 a $170 \mathrm{~cm}$ de íleo é medido em sentido proximal e seccionado com grampeador linear. O segmento de íleo é interposto de forma isoperistáltica no jejuno proximal previamente seccionado. Em seguida, são realizadas três enteroanastomoses: a primeira íleo-ileal próxima ao ceco; outra próxima ao ângulo duodenojejunal; a terceira íleo-jejunal. O procedimento pode também ser feito com interposição ileal na primeira porção do duodeno. Conclusão - Esta técnica demonstrou-se segura, com baixa morbidade e mortalidade, factível cirurgicamente pela via laparoscópica e passível de ser ofertada como opção operatória ao tratamento da diabetes mellitus tipo 2.

ABSTRACT - Introduction - There is an evidence that the best results in terms of resolution of diabetes in morbidly obese patients are achieved with bilio-pancreatic bypass, especially the duodenal switch. These operations are characterized by partial gastrectomy and the rapid transit of food into the distal ileum through derivation of a significant segment of small intestine. The idea of performing the technique presented here was based on these principles. Method - The procedures are usually performed laparoscopically, after establishment of a pneumoperitoneum at $12-15 \mathrm{mmHg}$ and introduction of six trocars. The patient is initially positioned in $30^{\circ}$ reverse Trendelenburg with the surgeon on the right side of the patient. The sleeve gastrectomy is performed using the anatomical distal trifurcation of the anterior vagus nerve as a reference. The devascularization of the greater curvature is performed and extends to the oesophagogastric junction. With an intra-gastric calibration tube of $20 \mathrm{~mm}$ positioned along the lesser curvature, gastric resection starts at the proximal antrum with linear stapler up to oesophagogastric angle. An invaginating running suture is also performed. To perform the ileal interposition in the proximal jejunum, it is divided $20-30 \mathrm{~cm}$ distally with a $45-\mathrm{mm}$ linear stapler. The cecum is identified and the distal ileum transected $30 \mathrm{~cm}$ proximal to the ileocecal valve. A 170 to $200 \mathrm{~cm}$ of ileum was measured proximally along the anti-mesenteric border using a $10-\mathrm{cm}$ marked atraumatic grasper, and transected with a $45-\mathrm{mm}$ linear stapler. This segment of ileum is interposed in an isoperistaltic way into the proximal jejunum, previously divided. Next are perform three side-to-side enteroanastomosis. The first enteroanastomosis is the ileo-ileostomy, then the jejuno-ileostomy and finally, the ileo-jejunostomy. All three mesenteric defects are closed with interrupted sutures. The procedure can also be done with ileal interposition upinto the duodenum. Conclusion - This technique was safe, with low morbidity and mortality, feasible through laparoscopy and possible to be offered as an option for surgical treatment of type 2 diabetes. 
INTRODUÇÃO

prevalência da diabetes mellitus tipo 2
(DM2) teve substancial aumento na década
passada nos Estados Unidos e em todo o mundo, e apresenta estreita correlação com o marcado incremento na prevalência da obesidade. $O$ principal fator de risco é a obesidade, e aproximadamente $90 \%$ dos portadores apresentam sobrepeso ou obesidade ${ }^{3,13,14}$.

Há evidências que os melhores resultados em termos de resolução da DM2 em pacientes obesos mórbidos são atingidos com as derivações biliopancreáticas, em especial o duodenal switch ${ }^{1,5,6,6,7,10}$. Essas operações caracterizam-se por redução do estômago através de gastrectomia e o rápido trânsito dos alimentos para o íleo distal, através de expressiva derivação de segmentos do intestino delgado.

A idéia da realização da técnica aqui apresentada baseou-se nestes princípios.

\section{TÉCNICA}

Os procedimentos são habitualmente realizados por via laparoscópica, apesar da abordagem laparotômica ser também possível e indicada.

Após estabelecimento de pneumoperitônio com 12 a $15 \mathrm{mmHg}$, seis trocárteres (três de $5 \mathrm{~mm}$, dois de $12 \mathrm{~mm}$ e um de $10 \mathrm{~mm}$ ) são introduzidos na cavidade abdominal. O paciente é inicialmente posicionado em proclive de $30^{\circ}$. O cirurgião coloca-se à direita do paciente.

Inicia-se a gastrectomia vertical com a ligadura dos vasos da grande curvatura gástrica tendo como referência anatômica a trifurcação distal do vago anterior. A desvascularização assim como a gastrectomia é iniciada na altura da "pata de ganso" nos pacientes com IMC entre 30 e 35. Para aqueles abaixo de 30, inicia-se de 2 a $4 \mathrm{~cm}$ acima da incisura angular. A desvascularização da grande curvatura é realizada com energia ultrassônica e/ou bipolar e se estende até o ângulo esofagogástrico. Passo seguinte é a introdução de um calibrador intra-gástrico de $20 \mathrm{~mm}$ posicionado ao longo da pequena curvatura gástrica até proximidades do piloro. A ressecção gástrica iniciase no antro, nos níveis mencionados e se estende até $\mathrm{o}$ ângulo esofagogástrico. Utiliza-se grampeador linear de 45 ou $60 \mathrm{~mm}$, carga verde ou azul. Ainda com o calibrador no interior do estômago, é feita uma sutura contínua, invaginante sobre o grampeamento, utilizando fio de polipropileno 3-0.

Interposição ileal na altura do jejuno proximal

Para a realização da interposição ileal na altura do jejuno proximal, o paciente é colocado em posição de Trendelenburg, $10^{\circ}$. O ângulo duodenojejunal é identificado e o jejuno seccionado de 20 a $30 \mathrm{~cm}$ à jusante com grampeador linear de $45 \mathrm{~mm}$, carga branca. O mesentério é aberto até sua base com energia ultrassônica ou bipolar. Em seguida, o cirurgião se desloca para o lado esquerdo do paciente. O ceco é identificado e o íleo distal seccionado a $30 \mathrm{~cm}$ no sentido proximal. $\mathrm{O}$ mesentério também é aberto até a sua base. Um segmento de 150 a $170 \mathrm{~cm}$ de íleo é medido em sentido proximal e seccionado com grampeador linear de $45 \mathrm{~mm}$, carga branca. Repete-se a abertura do mesentério. A medida é feita ao longo do bordo anti-mesentérico, utilizando uma pinça atraumática marcada em $10 \mathrm{~cm}$. O segmento de íleo é interposto de forma isoperistáltica no jejuno proximal previamente seccionado. Em seguida, são realizadas três enteroanastomoses. A primeira é íleo-ileal, próxima ao ceco. É realizada uma enterotomia de aproximadamente $1,0 \mathrm{~cm}$ em ambas as extremidades das alças e a anastomose é feita com uso de um grampeador linear com carga branca de $45 \mathrm{~mm}$. A enterotomia é fechada com sutura contínua, fio absorvível 3-0. 0 restante da linha de grampeamento é invaginada com o mesmo fio, sutura contínua. Em seguida, realiza-se o fechamento da primeira brecha mesentérica com pontos separados, fio de polipropileno 3-0. A segunda enteroanastomose, próxima ao ligamento duodenojejunal, é uma jejunoileostomia realizada da mesma maneira. A brecha mesentérica dessa anastomose também é fechada com pontos separados, fio inabsorvível. A terceira e última enteroanastomose é uma íleo-jejunostomia realizada conforme descrito anteriormente, assim como o fechamento da brecha mesentérica ${ }^{2,4,9,11,12,15,16}$.

\section{Interposição ileal no duodeno}

Para os casos de interposição ileal no duodeno, a desvascularização da grande curvatura gástrica se estende até 4 ou $5 \mathrm{~cm}$ além do piloro. O duodeno é seccionado com grampeador linear, carga azul de 45 ou $60 \mathrm{~mm}$. Uma sutura contínua com fio de polipropileno 3-0 é realizada sobre o grampeamento do coto duodenal. O reservatório gástrico e o segmento proximal do duodeno são transpostos para o abdome inferior através de abertura do mesocólon adjacente ao ligamento duodenojejunal. Um segmento de íleo distal é criado a $30 \mathrm{~cm}$ acima do ceco conforme descrito acima. Em seguida são realizadas três enteroanastomoses. A primeira é uma íleo-ileal. Em seguida, o segmento ileal é transposto e sua porção proximal é anastomosada de forma isoperistáltica ao duodeno previamente dividido. A segunda anastomose, duodeno-ileal, é feita manualmente em dois planos, pontos separados usando fio absorvível 3-0. A terceira anastomose, íleojejunal, é realizada após marcar-se um ponto $50 \mathrm{~cm}$. distal à jusante do ângulo duodenojejunal. É realizada uma anastomose látero-lateral entre a extremidade distal do íleo transposto e o jejuno. As duas aberturas do mesentério e a brecha da anastomose duodeno-ileal são fechadas conforme acima, assim como os orifícios dos trocárteres de $12 \mathrm{~mm}$ (Figuras 1 e 2). 


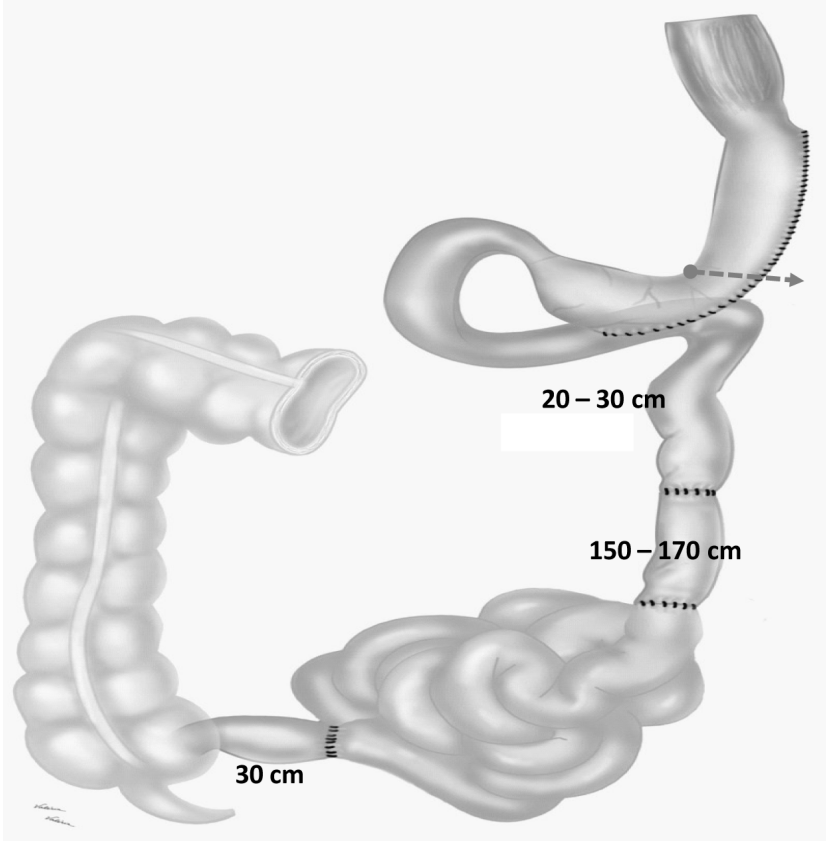

FIGURA 1 - Interposição Ileal - Gastrectomia Vertical

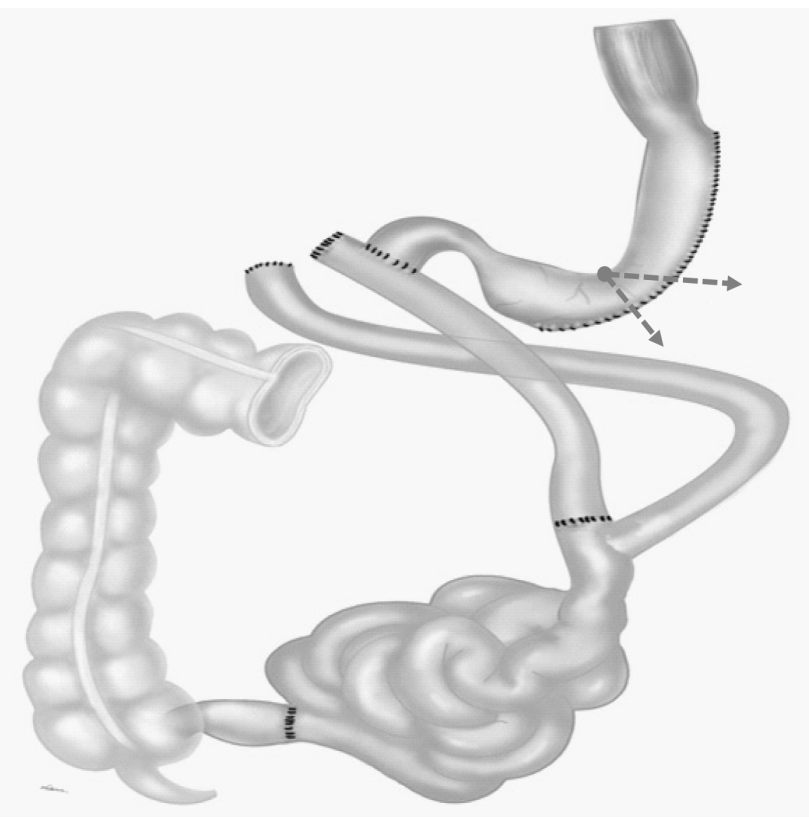

FIGURA 2 - Interposição - Gastrectomia Vertical Desviada

\section{CONCLUSÃO}

Esta técnica demonstrou-se segura, com baixa morbidade e mortalidade, factível cirurgicamente pela via laparoscópica e passível de ser ofertada como opção operatória ao tratamento da diabetes mellitus tipo 2 .

\section{REFERÊNCIAS}

1. Buchwald H, Avidor $Y$, Braunwald E, Jensen ME, Pories W, Fahrback K and Schoelles K. Bariatric Surgery: A Systematic Review and Meta-analysis. JAMA 2004; 292:1724-1737.

2. De Paula AL, Macedo AL, Prudente AS, Queiroz L, Schraibman V, Pinus J. Laparoscopic sleeve gastrectomy with ileal interposition ( ${ }_{\mu \prime \prime}$ neuroendocrine brake"'). Surg Obes Relat Dis. 2006;2:464-467.

3. De Paula AL, Macedo ALV, Prudente A, Silva L, Schraibman V, Neto JG, Pinus J, Cury EK, Szajnbok P, Dario RPD, Bertocco L, Diniz K, Gaudencio J, Gebin L, D"Orto U, Cison D, Penhavel F. Neuroendocrine brake for the treatment of morbid obesity:preliminary report. Einstein 2005;3:110-114.

4. De Paula AL, Stival AS, Macedo A, Ribamar J, Mancini M, Halpern A, Vencio S. Prospective randomized controlled trial comparing 2 versions of laparoscopic ileal interposition associated with sleeve gastrectomy for patients with type 2 diabetes with BMI $21-34 \mathrm{~kg} /$ m2. Surg Obes Relat Dis 2010;6:296-305.

5. De Paula, AL, Macedo AL, Rassi N, Vencio S, Machado CA, Mota BR, Silva LQ, Halpern A, Schraibman V. Laparoscopic treatment of metabolic syndrome in patients with type 2 diabetes mellitus. Surg Endosc 2008;22:2670-8.

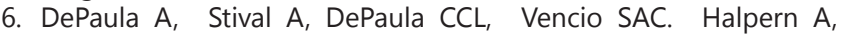
Impact On Dyslipidemia Of The Laparoscopic Ileal Interposition Associated To Sleeve Gastrectomy In Type 2 Diabetic Patients. J Gastrointest Surg 2010 (in press) JGSU-D-10-00295R1

7. DePaula AL, Halpern A, Muscelli E, Mari A, Stival A, Vencio V, Ferrannini E. Diabetes improvement following ileal interposition with sleeve gastrectomy in type 2 diabetic patients: mechanisms and relation to weight loss. Diabetologia 2009;Vol.52:S1.

8. DePaula AL, Macedo AL, Schraibman $V$, Mota $B R$, Vencio $S$. Hormonal evaluation following laparoscopic treatment of type 2 diabetes mellitus patients with BMI 20-34. Surg Endosc. 2009 Aug;23(8):1724-32.

9. DePaula AL, Macedo ALV, Mota BR, Schraibman V. Laparoscopic ileal interposition associated to a diverted sleeve gastrectomy is an effective operation for the treatment of type 2 diabetes mellitus patients with BMI 21-29. Surg Endosc 2009;23:1313-20.

10. DePaula AL, Macedo ALV, Rassi N, Machado CA, Schraibman V, Silva LQ, Halpern H. Laparoscopic treatment of type 2 diabetes mellitus for patients with a body mass index less than 35 . Surg Endosc 2008;22:706-16

11. Halpern A, DePaula AL, Stival AR, DePaula, CCL, Vencio SAC. Glycemic Variability Evaluated by the Continuous Glucose Monitoring System in Type 2 Diabetic Patients with BMI 24 - 34 Submitted to Ileal Interposition Associated with Sleeve Gastrectomy. Diabetes 2010;59:S1:A133

12. Hari Kumar KVS, Ugale S, Gupta N, Naik V, Kumar P, Bhaskar P, Modi KD. Ileal Interposition with Sleeve Gastrectomy for Control of Type 2 Diabetes. Diabetes Technol Ther. 2009;11:785-9.

13. Pories WJ. Bariatric Surgery: Risks and Rewards. J Clin Endocrinol Metab 2008;93:S89-S96.

14. Sjöström L, Narbro K, Sjöström CD, Karason K, Larsson B, Wedel H, Lystig T, Sullivan M, Bouchard C, Carlsson B. The Swedish Obese Subjects Study Effects of Bariatric Surgery on Mortality in Swedish Obese Subjects. N Engl J Med 2007;357:741-752.

15. Vencio SAC ,Stival AR, DePaula, CCL, Halpern A, DePaula AL. Evaluation of Insulin Sensitivity and Secretion in Type 2 Diabetic Patients with BMI below 35 Submitted to Ileal Interposition with Sleeve Gastrectomy using the Euglycaemic Hyperinsulinemic Clamp With IVGTT. Diabetes 2010;59:S1

16. Vencio SAC, Stival AR, DePaula CCL, Halpern A, DePaula AL. Early Mechanisms of Glucose Improvement Following Laparoscopic Ileal Interposition Associated to a Sleeve Gastrectomy Evaluated by the Euglycemic Hyperinsulinemic Clamp in Type 2 Diabetic Patients with BMI below 35. SOARD 2010;6:S22 